\title{
Broad Ligament Fibroid Mimicking as Ovarian Tumour
}

\author{
Leelavathi', Priyankur Roy², K. Triveni², S. Impana ${ }^{2}$ \\ ${ }^{1}$ Department of OBG Unit-IV, JSS Medical College \& Hospital, JSS University, Mysore, India \\ ${ }^{2}$ Department of Obstetrics and Gynaecology, JSS Medical College \& Hospital, Mysore, India \\ Email:drleelavathibasava@gmail.com, priyankurroy@hotmail.com, triveni.kondareddy@gmail.com, \\ impanaistarth@gmail.com
}

Received 5 August 2015; accepted 22 September 2015; published 25 September 2015

Copyright (C) 2015 by authors and Scientific Research Publishing Inc.

This work is licensed under the Creative Commons Attribution International License (CC BY).

http://creativecommons.org/licenses/by/4.0/

(c) () Open Access

\begin{abstract}
Background: Giant fibroids usually arise from the uterus, and very rarely can also arise from the broad ligament. Large fibroids can undergo hyaline, cystic degeneration and very rarely red degeneration. Case Report: In our case, ovarian neoplasm of the ovaries was suspected, as an adnexal mass with cystic degeneration and intervening septations were seen which were not separate from the lesion. The MRI findings were also characteristic of ovarian neoplasm. Giant fibroid with cystic degeneration was a rare differential diagnosis. Broad ligament fibroid excision, total abdominal hysterectomy, and bilateral salpingo-oophorectomy were performed on the patient. The diagnosis was confirmed on histopathological examination. Conclusion: Broad ligament leiomyoma should be kept as an important differential diagnosis for solid adnexal or ovarian masses.
\end{abstract}

\section{Keywords}

Broad Ligament Fibroid, Magnetic Resonance Imaging, Ovarian Neoplasm, Ultrasonography

\section{Introduction}

Fibroid is the commonest of all uterine tumours, most commonly seen during the reproductive age group. It is a benign smooth muscle tumour. It can be intrauterine or extrauterine. The classification is determined by its site of origin and direction of growth. They are broadly classified into three main groups: subserous, interstitial, and submucous. Entities called extra-uterine fibroids exist but they are not as common as uterine fibroids.

Extra-uterine fibroids may develop in the broad ligament or at other sites where smooth muscle exists [1]. Primary tumours of broad ligament are rare. Broad ligament tumours pose specific diagnostic difficulties because of their rarity. The tumours known to occur in the broad ligament are epithelial and mesenchymal tu- 
mours.

Menstrual disturbances and dysmenorrhea are the commonest symptoms of fibroids. Symptoms related to pressure caused by the mass are also seen in certain cases [2]. Degeneration, infection, haemorrhage, and necrosis are the most common secondary changes noted. Rarely, sarcomatous changes are also seen.

About 1 out of 1000 lesions becomes malignant, typically as a leiomyosarcoma on histology. A sign that a lesion may be malignant is growth after menopause. There is no consensus among pathologists regarding the transformation of leiomyoma into a sarcoma.

\section{Case Report}

A 48-year-old multiparous lady, married for 30 years, last child birth 25 years back, tubectomised, came with complaints of backache and heaviness in the lower abdomen for the past 6 months. She had attained menopause 6 years back. She was a known case of Hypertension on treatment. She had no history of bowel or bladder disturbances, weight loss, anorexia, white discharge or bleeding per vaginum. Her previous menstrual cycles were regular.

On examination, the patient was afebrile. She was obese (BMI was $34.2 \mathrm{~kg} / \mathrm{m}^{2}$ ). Her vital signs were stable. No mass was palpable on abdominal examination. On speculum examination, cervix could not be visualized, and no abnormal discharge was seen. On vaginal examination, a mass was felt in the right fornix of size approximately $7 \times 6 \mathrm{cms}$; bulky, regular uterus could be made out separately.

Routine pre-operative blood investigations were within normal limits except for her Hepatitis B status being positive. Serum CA-125 titre was $16 \mathrm{~m} \mathrm{IU/L}$. On ultrasonography, a large well-defined, heteroechoic mass lesion measuring $8.5 \times 7.8 \times 8.2 \mathrm{cms}$ was seen in the right adnexa-most likely to be ovarian mass/fibroid; minimal fluid was seen in the pouch of douglas; right ovary was not seen separately from the mass; left ovary was normal. A probable diagnosis of right ovarian mass was made. On Magnetic Resonance Imaging (MRI) of abdomen and pelvis, a large mixed density abdomino-pelvic lesion was seen in the right adnexa with cystic changes/areas of degeneration; right ovary could not be seen separately. The MRI appearance was typical of cystic ovarian neoplasm. Final diagnosis based on the MRI of abdomen and pelvis was right ovarian neoplasm.

The patient was taken up for an exploratory laparotomy after a provisional diagnosis of malignant ovarian tumour was made. Intra-operatively, an abdomino-pelvic mass in the right side of size approximately $8 \times 7 \times 5$ cms was seen arising adjacent to the uterus in between the folds of the broad ligament pushing the ureter medially. Bilateral tubes and ovaries were normal. As the tumour was distorting the anatomy, careful dissection was done to prevent ureteric injuries. Excision of tumour with total abdominal hysterectomy and bilateral salpingooophorectomy was performed and the specimen was sent for histopathological examination. Post-operative course was uneventful.

The mass in the broad ligament showed interlacing bundles of smooth muscle cells scattered thick-walled blood vessels and evidence of cystic degeneration. On staining with haematoxylin and eosin, the endo-myometrium was composed of round to oval glands lined by columnar epithelium set in a compact stroma. Thus, a final diagnosis of leiomyoma was made (Figure 1, Figure 2).

\section{Discussion}

Fibroids, may become adherent to surrounding structures like the omentum or the broad ligament and subsequently develop an auxiliary blood supply and lose their original uterine attachment. A critical literature review states that fibroids may be adherent to the broad ligament and originate from the smooth muscle elements of that ligament which are hormone sensitive. Extra-uterine pelvic masses can compress the surrounding structures and produce symptoms of urinary outflow obstruction. On ultrasound, a typical leiomyoma usually has a whorled appearance, with variable echogenicity depending on the extent of degeneration and calcification [3].

Broad ligament leiomyoma can originate from the uterus and invade the broad ligament or it can originate from broad ligament itself. These benign tumours are usually asymptomatic. However, if the leiomyoma reaches significant size, it can push uterus to contralateral side or it can potentially compress the surrounding pelvis structure and manifest clinically with various sign and symptoms. The location of tumours often determines the various symptoms [4]. It is thus, important for any adnexal mass to be discriminated between benign and malignant nature pre-operatively for optimal patient management. The differential diagnosis for broad ligament leiomyoma includes masses from ovarian origin — benign or malignant, broad ligament cyst, lymphadenopathy and 


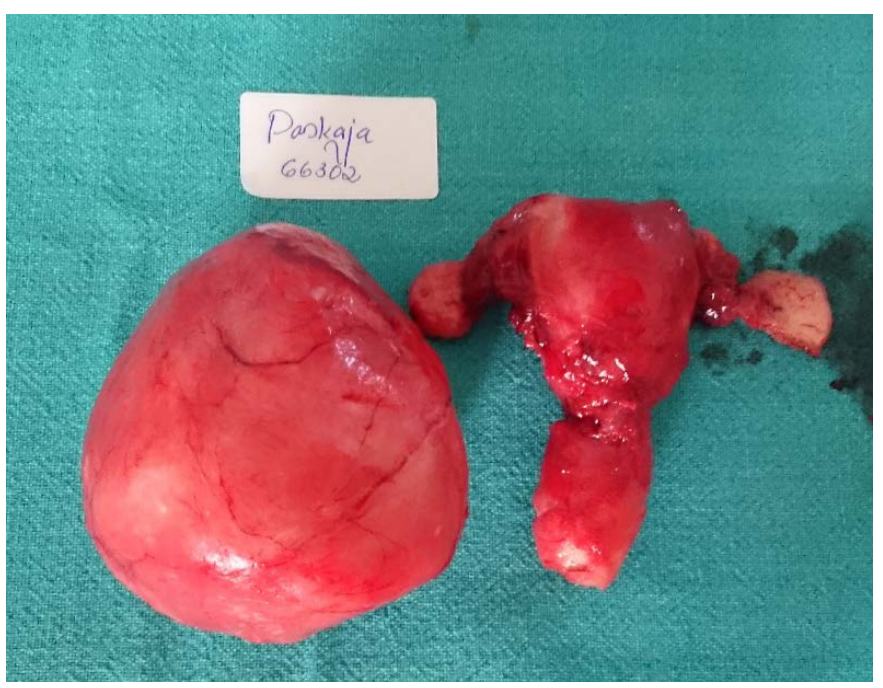

Figure 1. Gross operative specimen of uterus, cervix, ovaries and broad ligament fibroid.

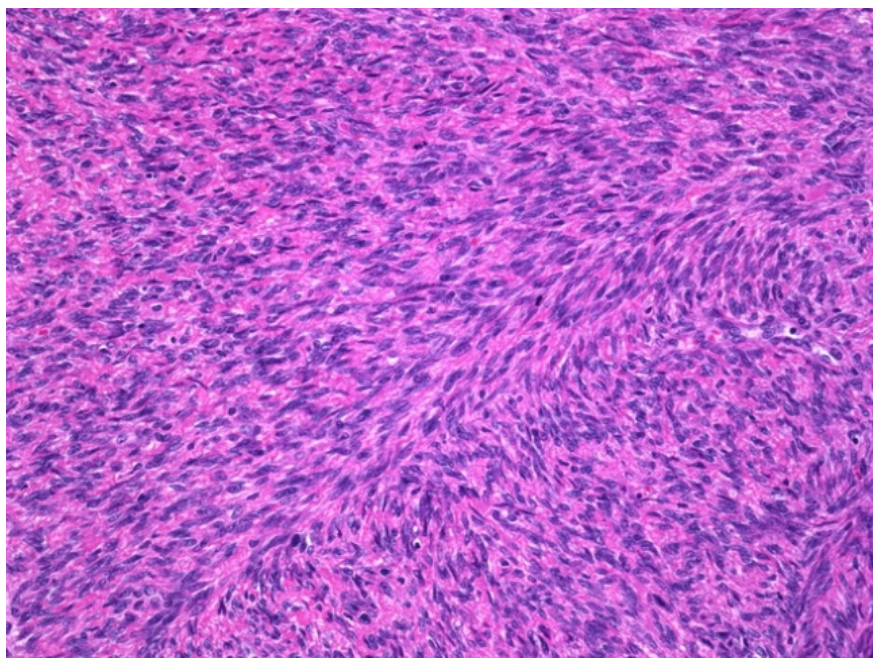

Figure 2. Histopathological Examination, H \& E stain: Concentrically arranged smooth muscle cells - Leiomyoma.

tubo-ovarian masses.

In our case, on clinical and radiological investigation it was suspicious of ovarian neoplasm. The serum levels of cancer antigen CA-125 was within normal range. Elevated CA-125 levels may point to metastatic ovarian malignancies, but it can also be raised in endometriosis, endometriomas and serous benign tumours [5] which make diagnosis difficult preoperatively. Histopathology plays an important role in diagnosis for such cases.

Leiomyomas may be single or multiple. In our case, it was a large true broad ligament fibroid. Broad ligament leiomyomas have the potential to grow to large size [6]. Secondary changes in leiomyomas may occur and the most common are degeneration, infection, haemorrhage and necrosis. The cystic changes in lesion mimic the metastatic malignant ovarian tumours [7]. Primary leiomyosarcoma in broad ligament is very rarely reported [8]. Hence, proper histopathological evaluation is important for patients' further management.

Leiomyomas could also be confused with other tumours of the retroperitoneum, omentum and mesenteries. GISTs occur in the entire gastrointestinal tract and originate from gastrointestinal pacemaker cells (Cajal's interstitial cells). Diagnosis of GIST could be deceptive because of their similarity in appearance to gynaecological neoplasms. It is crucial to separate GISTs from possible misdiagnosis because their prognosis and treatment could be clearly differ [9]. 


\section{Conclusion}

A major diagnostic challenge of extra-uterine fibroid is that it can often mimic malignant tumours at imaging. Fibroids are usually histologically benign, but their management can be challenging, especially when it is extra-uterine. Extra-uterine leiomyomas mimics ovarian tumours on clinical and radiological examination. Pedunculated leiomyomas should also be considered in the differential diagnosis of a multi-locular and predominantly cystic adnexal mass.

\section{References}

[1] Kumar, P. and Malhotra, N. (2008) Jeffcoate’s Principles of Gynaecology. 7th Edition, Jaypee Brothers, New Delhi, 492.

[2] Barek, J.S. (2007) Novack’s Gynaecology. 15th Edition, Lippincott Williams and Wilkins, Wolters Kluwer (India), New Delhi, 470.

[3] Fasih, N., Prasad Shanbhogue, A.K., Macdonald, D.B., Fraser-Hill, M.A., Papadatos, D. and Kielar, A.Z. (2008) Leiomyomas beyond the Uterus: Unusual Locations, Rare Manifestations. Radiographics, 28, 1931-1948. http://dx.doi.org/10.1148/rg.287085095

[4] Stewart, E.A. (2001) Uterine Fibroids. The Lancet, 357, 293-298. http://dx.doi.org/10.1016/S0140-6736(00)03622-9

[5] Jacobs, I. and Bast Jr., R.C. (1989) The CA 125 Tumour Associated Antigen: A Review of the Literature. Human Reproduction, 4, 1-12.

[6] Godbole, R.R., Laksmi, K.S. and Vasant, K. (2012) Rare Case of Giant Broad Ligament Fibroid with Myxoid Degeneration. Journal of the Scientific Society, 39, 144-146. http://dx.doi.org/10.4103/0974-5009.105921

[7] Rajanna, D.K., Pandey, V., Janardhan, S. and Datti, S.N. (2013) Broad Ligament Fibroid Mimicking as Ovarian Tumour on Ultrasonography and Computed Tomography Scan. Journal of Clinical Imaging Science, 3, 8. http://dx.doi.org/10.4103/2156-7514.107912

[8] Duhan, N., Singh, S., Kadian, Y.S., Duhan, U., Rajotia, N. and Sangwan, N. (2009) Primary Leiomyosarcoma of the Broad Ligament. A Case Report and Review of Literature. Archives of Gynecology and Obstetrics, 279, 705-708. http://dx.doi.org/10.1007/s00404-008-0777-2

[9] Karaca, N., Akpak, Y.K., Tatar, Z., Batmaz, G. and Erken, A. (2016) Gastrointestinal Stromal Tumour: May Mimic Adnexal Mass. Global Journal of Health Science, 8, 20. 